\title{
First-order EQ-logic
}

\author{
Martin Dyba, Vilém Novák \\ Centre of Excellence IT4Innovations, Division of the University of Ostrava \\ Institute for Research and Applications of Fuzzy Modeling \\ 30. dubna 22, 70200 Ostrava, Czech Republic
}

\begin{abstract}
This paper represents the third step in the development of EQ-logics. Namely, after developing propositional and higher-order EQ-logics, we focus also on predicate one. First, we give a brief overview of the propositional EQ-logic and then develop syntax and semantics of predicate EQ-logic. Finally, we prove completeness by constructing a model of a consistent theory of EQ-logic from the syntactical material, as usual.
\end{abstract}

Keywords: EQ-algebra; EQ-logic; mathematical fuzzy logic;

\section{Introduction}

This paper is the third step in the development of EQ-logics as special kinds of fuzzy logics based on EQ-algebras. Recall that the latter are algebras in which the basic operation is fuzzy equality (i.e., interpretation of equivalence) instead of residuation (i.e., interpretation of implication). Till now, propositional EQ-logic [1] and EQ-algebrabased higher-order fuzzy logic (fuzzy type theory) [2] have been developed. Therefore, it is interesting to complete the picture also by first-order EQlogic. Let us emphasize that the strong conjunction \& (interpreted the multiplication $\otimes$ ) in EQ-logics is non-commutative.

In this paper, we will show, how EQ-logic with complete syntax can be developed. The proof proceeds in the standard way by constructing a model from the considered syntactical material. To do it, however, several special questions had to be answered and elaborated in detail. Besides others, we had to decide, what properties of the considered EQ-algebra are necessary to be able to construct a model of a theory in EQ-logic. We found that we need the $\Delta$-operation, linearity, and also require fulfilling of the inequality $\Delta(a \sim b) \leq a \otimes c \sim b \otimes c$.

\section{EQ-logic: An overview}

In this section, we will briefly overview basic definitions and the main properties of EQ-algebras and basic propositional EQ-logics.

\subsection{EQ-algebra}

The concept of EQ-algebra appeared for the first time in [3] and elaborated in more detail in $[4,5]$.

\section{Definition 1}

A non-commutative $E Q$-algebra $\mathcal{E}$ is an algebra

$$
\mathcal{E}=\langle E, \wedge, \otimes, \sim, \mathbf{1}\rangle
$$

of type $(2,2,2,0)$ fulfilling the following axioms for all $a, b, c, d \in E$ :

(E1) $\langle E, \wedge, \mathbf{1}\rangle$ is a commutative idempotent monoid (i.e. $\wedge$-semilattice with top element 1). We put $a \leq b$ iff $a \wedge b=a$, as usual.

(E2) $\langle E, \otimes, \mathbf{1}\rangle$ is a monoid and $\otimes$ is isotone w.r.t. $\leq$.

(E3) $a \sim a=1$

(E4) $((a \wedge b) \sim c) \otimes(d \sim a) \leq c \sim(d \wedge b)$

(E5) $(a \sim b) \otimes(c \sim d) \leq(a \sim c) \sim(b \sim d)$

(E6) $(a \wedge b \wedge c) \sim a \leq(a \wedge b) \sim a$

(E7) $a \otimes b \leq a \sim b$

The operation $\sim$ is fuzzy equality, $\wedge$ is meet and $\otimes$ is multiplication. An EQ-algebra is commutative if $\otimes$ is a commutative operation.

A derived operation

$$
a \rightarrow b=(a \wedge b) \sim a
$$

where $a, b \in E$ is called implication.

If $\mathcal{E}$ contains also the bottom element $\mathbf{0}$ then we put

$$
\neg a=a \sim \mathbf{0}, \quad a \in E
$$

and call $\neg a$ a negation of $a \in E$.

\section{Definition 2}

Let $\mathcal{E}$ be an $E Q$-algebra and $a, b, c, d \in E$. We say that $\mathcal{E}$ is:

(i) separated if $a \sim b=\mathbf{1}$ implies $a=b$.

(ii) $\operatorname{good}$ if $a \sim \mathbf{1}=a$.

(iii) prelinear if for all $a, b \in E, \sup \{a \rightarrow b, b \rightarrow$ $a\}=1$.

(iv) lattice EQ-algebra ( $\ell E Q$-algebra) if it is a lattice and for all $a, b, c, d \in E$,

(E8) $((a \vee b) \sim c) \otimes(d \sim a) \leq(d \vee b) \sim c$. 
Theorem 1 ([6])

If a good EQ-algebra $\mathcal{E}$ satisfies

$$
(a \rightarrow b) \vee(d \rightarrow(d \otimes(c \rightarrow(b \rightarrow a) \otimes c)))=\mathbf{1}
$$

for all $a, b, c, d \in E$ then it is prelinear and representable, i.e. it is isomorphic to a subdirect product of linearly ordered EQ-algebras.

\subsection{Basic EQ-logic}

The basic EQ-logic was introduced in [1]. It has three basic binary connectives $\wedge, \&, \equiv$ and the truth constant $T$. Implication is a derived connective defined by

$$
A \Rightarrow B:=(A \wedge B) \equiv A .
$$

The algebra of truth values for basic EQ-logic is formed by a good non-commutative EQ-algebra. The following formulas are its logical axioms:

(EQ1) $(A \equiv \top) \equiv A$

(EQ2) $A \wedge B \equiv B \wedge A$

(EQ3) $(A \bigcirc B) \bigcirc C \equiv A \bigcirc(B \bigcirc C), \quad \bigcirc \in\{\wedge, \&\}$

(EQ4) $A \wedge A \equiv A$

(EQ5) $A \wedge \top \equiv A$

(EQ6) $A \& \top \equiv A$

(EQ7) $\top \& A \equiv A$

(EQ8a) $((A \wedge B) \& C) \Rightarrow(B \& C)$

(EQ8b) $(C \&(A \wedge B)) \Rightarrow(C \& B)$

(EQ9) $((A \wedge B) \equiv C) \&(D \equiv A) \Rightarrow(C \equiv(D \wedge B))$

(EQ10) $(A \equiv B) \&(C \equiv D) \Rightarrow(A \equiv C) \equiv(D \equiv B)$

(EQ11) $(A \Rightarrow(B \wedge C)) \Rightarrow(A \Rightarrow B)$

The inference rules of basic EQ-logic are equanimity rule (EA) (from $A$ and $A \equiv B$ derive $B$ ) and Leibniz rule (Leib) (from $A \equiv B$ derive $C[\mathbf{p}:=$ $A] \equiv C[\mathbf{p}:=B]$ where the expression of the form $C[\mathbf{p}:=X]$ for $X:=A$ or $X:=B$ denotes a formula resulting from $C$ by replacing all occurrences of the variable $\mathbf{p}$ in $C$ by the formula $X$ ).

\section{Lemma 1 ([1])}

(a) $\vdash A \equiv A$,

(b) $\vdash(A \Rightarrow B) \Rightarrow((A \wedge C) \Rightarrow B))$,

(c) $A, A \Rightarrow B \vdash B$,

(Modus Ponens)

(d) $A, B \vdash A \& B$,

(e) $\vdash(A \equiv B) \equiv(B \equiv A)$,

(f) $\vdash(A \equiv B) \Rightarrow(A \Rightarrow B)$,

(g) $\vdash(A \Rightarrow B) \&(B \Rightarrow A) \Rightarrow(A \equiv B)$,

(h) $\vdash(A \equiv B) \&(B \equiv C) \Rightarrow(A \equiv C)$, (i) $\vdash(A \&(A \equiv B)) \Rightarrow B$,

(j) $\vdash(A \Rightarrow B) \&(B \Rightarrow C) \Rightarrow(A \Rightarrow C)$,

(k) $\vdash A \& B \Rightarrow A \equiv B$,

(l) $\vdash(C \Rightarrow A) \&(C \Rightarrow B) \Rightarrow(C \Rightarrow(A \wedge B))$,

$(m) \vdash((A \equiv B) \&(C \equiv D)) \Rightarrow((A \wedge C) \equiv(B \wedge D))$,

(n) $\vdash(A \Rightarrow B) \Rightarrow((B \Rightarrow C) \Rightarrow(A \Rightarrow C))$,

(o) $\vdash(A \Rightarrow(B \Rightarrow C)) \Rightarrow(B \Rightarrow(A \Rightarrow C))$.

\section{Theorem 2 (Completeness)}

The following is equivalent for every formula $A$ :

(a) $\vdash A$,

(b) $e(A)=\mathbf{1}$ for every good non-commutative EQalgebra $\mathcal{E}$ and a truth evaluation $e: F_{J} \longrightarrow E$.

For more details and other kinds of EQ-logic see [1].

\section{3. $\mathrm{EQ}_{\Delta}$-logic}

This logic is obtained from the basic one by enriching it by a $\Delta$ connective.

\subsection{Lattice $\mathrm{EQ}_{\Delta}$-algebra}

Lattice $\mathrm{EQ}_{\Delta}$-algebras form one of important classes of EQ-algebras. Algebras from this class are used as structures of truth values for prelinear $\mathrm{EQ}_{\Delta}$-logic.

\section{Definition 3}

$A$ lattice $E Q_{\Delta}$-algebra ( $\ell E Q_{\Delta}$-algebra) is an algebra $\mathcal{E}_{\Delta}=\langle E, \wedge, \vee, \otimes, \sim, \Delta, \mathbf{0}, \mathbf{1}\rangle$ where $\langle E, \wedge, \vee, \otimes$, $\sim, \mathbf{0}, \mathbf{1}\rangle$ is a good non-commutative and bounded $\ell E Q$-algebra ( $\mathbf{0}$ and $\mathbf{1}$ are bottom and top elements, respectively) expanded by a unary operation $\Delta: E \longrightarrow E$ fulfilling the following axioms:

$(E \Delta 1) \Delta \mathbf{1}=\mathbf{1}$

$(E \Delta 2) \quad \Delta a \leq \Delta \Delta a$

$(\mathrm{E} \Delta 3) \quad \Delta(a \sim b) \leq \Delta a \sim \Delta b$

$(E \Delta 4) \Delta(a \wedge b)=\Delta a \wedge \Delta b$

$(E \Delta 5) \quad \Delta a=\Delta a \otimes \Delta a$

$(E \Delta 6) \Delta(a \sim b) \leq(a \otimes c) \sim(b \otimes c)$

$(E \Delta 7) \Delta(a \sim b) \leq(c \otimes a) \sim(c \otimes b)$

$(E \Delta 8) \Delta(a \vee b) \leq \Delta a \vee \Delta b$

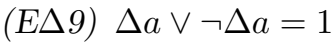

\section{Remark 1}

Axioms of the lattice $E Q_{\Delta}$-algebra are motivated by Novák's definition of the delta operation in EQalgebras (see [2]), where it was introduced for the first time. Axioms $(E \Delta 6)$ and $(E \Delta 7)$ guarantee good behavior of the multiplication with respect to the crisp equality. It follows from the results of [4] that if we omit $\Delta$ in $(E \Delta 6)$ and $(E \Delta 7)$ then the resulting $E Q$-algebra becomes residuated. 
As shown in the following lemma, two substitution axioms can be replaced by the only one in prelinear and good $\ell$ EQ-algebra.

\section{Lemma 2}

Let $\mathcal{E}$ be a prelinear and good $\ell E Q$-algebra. Then the substitution axioms (E4) and (E8) are equivalent to the following one:

$$
\text { (E9) } \begin{aligned}
((((a \wedge b) \vee c) \sim d) \otimes(f \sim c)) & \otimes(e \sim a) \leq \\
d & \sim(f \vee(b \wedge e))
\end{aligned}
$$

The following theorem characterizes a representable class of $\ell \mathrm{EQ}_{\Delta}$-algebras.

\section{Theorem 3 ([7])}

Let $\mathcal{E}_{\Delta}$ be $\ell E Q_{\Delta}$-algebra. Then the following properties are equivalent:

(a) $\mathcal{E}_{\Delta}$ is subdirectly embeddable into a product of linearly ordered $\ell E Q_{\Delta}$-algebras (i.e., $\mathcal{E}_{\Delta}$ is representable).

(b) $\mathcal{E}_{\Delta}$ satisfies condition (2) for all $a, b, c, d \in E$.

\subsection{Prelinear $\mathrm{EQ}_{\Delta}$-logic}

This logic is interesting because stronger form of the completeness theorem holds in it. Moreover, this logic will become the basis for the development of the predicate EQ-logic. The language of this logic is that of the basic EQ-logic extended by the binary connective $\mathrm{V}$, unary connective $\boldsymbol{\Delta}$ and logical constant $\perp$ (falsum). We also extend the language by the short

$$
\neg A:=A \equiv \perp .
$$

Formula (3) is definition of negation in this logic.

Semantics of this logic is formed by a noncommutative $\ell \mathrm{EQ}_{\Delta}$-algebra in which condition (2) is satisfied.

The complete list of logical axioms of the prelinear $\mathrm{EQ}_{\Delta}$-logic is the following:

$$
\begin{aligned}
& \text { (EQ1) }(A \equiv \top) \equiv A \\
& \text { (EQ2) } A \wedge B \equiv B \wedge A \\
& \text { (EQ3) }(A \bigcirc B) \bigcirc C \equiv A \bigcirc(B \bigcirc C), \quad \bigcirc \in\{\wedge, \boldsymbol{\&}\} \\
& \text { (EQ4) } A \wedge A \equiv A \\
& \text { (EQ5) } A \wedge \top \equiv A \\
& \text { (EQ6) } A \& \top \equiv A \\
& \text { (EQ7) } \top \& A \equiv A \\
& \text { (EQ8a) }((A \wedge B) \& C) \Rightarrow(B \& C) \\
& \text { (EQ8b) }(C \&(A \wedge B)) \Rightarrow(C \& B) \\
& \text { (EQ9) }((((A \wedge B) \vee C) \equiv D) \&(F \equiv C)) \& \\
& (E \equiv A) \Rightarrow(D \equiv(F \vee(B \wedge E))) \\
& (\mathrm{EQ} 10)(A \equiv B) \&(C \equiv D) \Rightarrow(A \equiv C) \equiv(D \equiv B)
\end{aligned}
$$

(EQ11) $(A \Rightarrow(B \wedge C)) \Rightarrow(A \Rightarrow B)$

$(\mathrm{EQ12})(A \vee B) \vee C \equiv A \vee(B \vee C)$

(EQ13) $A \vee(A \wedge B) \equiv A$

(EQ14) $(A \wedge \perp) \equiv \perp$

(EQ15) $(A \Rightarrow B) \vee$

$$
(D \Rightarrow(D \&(C \Rightarrow((B \Rightarrow A) \& C))))
$$

(EQ $\Delta 1) \Delta A \Rightarrow \Delta \Delta A$

$(\mathrm{EQ} \Delta 2) \boldsymbol{\Delta}(A \equiv B) \Rightarrow(\boldsymbol{\Delta} A \equiv \Delta B)$

$(\mathrm{EQ} \Delta 3) \Delta(A \wedge B) \equiv(\Delta A \wedge \Delta B)$

$(\mathrm{EQ} \Delta 4) \Delta A \equiv(\Delta A \& \Delta A)$

$(\mathrm{EQ} \Delta 5) \boldsymbol{\Delta}(A \equiv B) \Rightarrow((A \& C) \equiv(B \& C))$

$(\mathrm{EQ} \Delta 6) \boldsymbol{\Delta}(A \equiv B) \Rightarrow((C \& A) \equiv(C \& B))$

$(\mathrm{EQ} \Delta 7) \boldsymbol{\Delta}(A \vee B) \Rightarrow(\Delta A \vee \Delta B)$

$(\mathrm{EQ} \Delta 8) \boldsymbol{\Delta} A \vee \neg \Delta A$

\section{Remark 2}

Axioms of the basic EQ-logic are extended by axioms (EQ12)-(EQ14) which reflect the joinsemilattice structure. Moreover, axiom (EQ15) stands for the prelinearity and axiom (EQ9) express a common substitution axiom both for $\wedge$ and for $\vee$ and thus it replaces the original substitution axioms in EQ-logics. Finally, the $\Delta$-axioms correspond to the $\Delta$-axioms of the lattice $E Q_{\Delta}$-algebra.

Inference rules of the prelinear $\mathrm{EQ}_{\Delta}$-logic are those of the basic EQ-logic and the necessitation rule:

$$
\text { (N) } \frac{A}{\Delta A} \text {. }
$$

The main properties of the prelinear $\mathrm{EQ}_{\Delta}$-logic, with emphasize to the disjunction connective are introduced in the following lemma. Notice that the substitution property of both $\wedge($ Lemma $(3 \mathrm{~d}))$ and $\checkmark$ (Lemma $(3 \mathrm{~b}))$ is provable. Lemma 4 then shows properties of the delta connective in the prelinear $\mathrm{EQ}_{\Delta}$-logic.

\section{Lemma $3([7])$}

(a) $\vdash A \vee B \equiv B \vee A$,

(b) $\vdash((A \vee B) \equiv C) \&(D \equiv A) \Rightarrow((D \vee B) \equiv C)$,

(c) $\vdash A \vee \perp \equiv A$,

(d) $\vdash((A \wedge B) \equiv C) \&(D \equiv A) \Rightarrow(C \equiv(D \wedge B))$,

(e) $\vdash(A \Rightarrow B) \vee(B \Rightarrow A)$,

(f) $\vdash \top \vee A$,

(g) $\vdash A \Rightarrow(A \vee B)$,

(h) $\vdash A \wedge(A \vee B) \equiv A$, 


$$
\begin{aligned}
& \text { (i) } \vdash((A \vee B) \equiv B) \equiv(A \Rightarrow B), \\
& (\mathrm{j}) \vdash(A \Rightarrow C) \&(B \Rightarrow C) \Rightarrow((A \vee B) \Rightarrow C), \\
& (\mathrm{k}) \vdash((A \vee B) \Rightarrow C) \Rightarrow(A \Rightarrow C), \\
& (\mathrm{l}) \vdash(A \Rightarrow B) \Rightarrow(A \Rightarrow(B \vee C)), \\
& (\mathrm{m}) \vdash(A \vee B) \equiv(((A \Rightarrow B) \Rightarrow B) \wedge((B \Rightarrow A) \Rightarrow \\
& \quad A)), \\
& (\mathrm{n}) \vdash(A \equiv B) \Rightarrow((A \vee C) \equiv(B \vee C)), \\
& (\text { o } \vdash(A \equiv B) \&(C \equiv D) \Rightarrow((A \vee C) \equiv(B \vee D)) .
\end{aligned}
$$

\section{Lemma 4 ([7])}

(a) $\vdash \Delta A \Rightarrow A$,

(b) $\vdash \Delta(A \Rightarrow B) \Rightarrow(\Delta A \Rightarrow \Delta B)$,

(c) $\vdash \boldsymbol{\Delta}(\neg A) \Rightarrow \neg(\Delta A)$,

(d) $\vdash \boldsymbol{\Delta}(A \vee B) \equiv(\Delta A \vee \Delta B)$,

(e) $\vdash(\Delta A \& \Delta(A \Rightarrow B)) \Rightarrow \Delta B$,

(f) $\vdash \boldsymbol{\Delta}((A \equiv B) \&(C \equiv D)) \Rightarrow$

$$
((A \& C) \equiv(B \& D)) .
$$

\section{Theorem 4 (Completeness)}

For every formula $A \in F_{J}$ and for every theory $T$ the following is equivalent:

(a) $T \vdash A$.

(b) $e(A)=\mathbf{1}$ for every truth evaluation $e: F_{J} \longrightarrow$ $E$ and every linearly ordered, $\ell E Q_{\Delta}$-algebra $\mathcal{E}_{\Delta}$.

(c) $e(A)=\mathbf{1}$ for every truth evaluation $e: F_{J} \longrightarrow$ $E$ and every $\ell E Q_{\Delta}$-algebra $\mathcal{E}_{\Delta}$ satisfying condition (2).

\section{First-order EQ-logic}

In this section, we will introduce first-order EQlogic. After detailed analysis it turned out that this logic can be developed only on the basis of the prelinear $\mathrm{EQ}_{\Delta}$-logic. Moreover, its EQ-algebra of truth values must be linearly ordered.

\subsection{Syntax}

\section{Definition 4}

The language $J$ of predicate EQ-logic consists of:

(i) Object variables $x, y, \ldots$

(ii) Set of object constants Const $=\{\mathbf{u}, \mathbf{v}, \ldots\}$

(iii) Non-empty set of $n$-ary predicate symbols Pred $=\{P, Q, \ldots\}$

(iv) Logical (truth) constants $\top$ and $\perp$.

(v) Binary connectives $\wedge, \vee, \&$, $\equiv$ and unary connective $\Delta$.

(vi) Quantifiers $\forall, \exists$. (vii) Auxiliary symbols: brackets.

Terms are object variables and object constants.

Formulas of predicate EQ-logic are defined as follows:

\section{Definition 5}

(i) If $P$ is an $n$-ary predicate symbol and $t_{1}, \ldots, t_{n}$ are terms then $P\left(t_{1}, \ldots, t_{n}\right)$ is atomic formula.

(ii) Logical constants $\top$ and $\perp$ are formulas.

(iii) If $A, B$ are formulas then $A \wedge B, A \vee B$, $A \& B, A \equiv B, \Delta A$ are formulas.

(iv) If $A$ is formula and $x$ is an object variable then $(\forall x) A,(\exists x) A$ are formulas.

The set of all the well-formed formulas for the language $J$ is denoted by $F_{J}$ (we will also speak about J-formulas). The concepts of bound and free variables and open and closed formulas are classical.

Let $A(x)$ be a formula and $t$ a term. By $A_{x}[t]$ we denote a formula in which all the free occurrences of the variable $x$ are replaced by the term $t$. The concepts of substitutable term, scope of quantifiers, instance of a formula, closure are defined classically.

\subsection{Semantics}

Let $J$ be a language of the predicate EQ-logic and let $\mathcal{E}=\langle E, \wedge, \vee, \otimes, \sim, \Delta, \mathbf{0}, \mathbf{1}\rangle$ be a noncommutative linearly ordered $\ell \mathrm{EQ}_{\Delta}$-algebra. The $\mathcal{E}$-structure for $J$ is

$$
\mathcal{M}^{\mathcal{E}}=\left\langle M, \mathcal{E},\left\{r_{P}\right\}_{P \in \text { Pred }},\left\{m_{\mathbf{u}}\right\}_{\mathbf{u} \in \text { Const }}\right\rangle
$$

where $M$ is a nonempty set (domain), $r_{P}: M^{n} \longrightarrow$ $E$ is an n-ary fuzzy relation assigned to each predicate symbol $P$ and $m_{u} \in M$ is an element assigned to each object constant $\mathbf{u}$. An evaluation of object variables is a mapping $v$ assigning to each object variable $x$ an element $v(x) \in M$. If $v$ is an evaluation then by $v^{\prime}=v \backslash x$ we denote an evaluation which differs from $v$ in the variable $x$ only.

Interpretation of terms in $\mathcal{E}$-structure $\mathcal{M}^{\mathcal{E}}$ is defined as follows:

$$
\begin{gathered}
\mathcal{M}_{v}^{\mathcal{E}}(\mathbf{u})=m_{\mathbf{u}}, \quad \mathbf{u} \in J \\
\mathcal{M}_{v}^{\mathcal{E}}(x)=v(x)
\end{gathered}
$$

where $v$ is an evaluation of variables.

\section{Definition 6}

Let $\mathcal{M}^{\mathcal{E}}$ be a structure for the language $J$. We 
define interpretation $\mathcal{M}_{v}^{\mathcal{E}}$ of formulas as follows:

$$
\begin{gathered}
\mathcal{M}_{v}^{\mathcal{E}}\left(P\left(t_{1}, \ldots, t_{n}\right)\right)=r_{P}\left(\mathcal{M}^{\mathcal{E}}\left(t_{1}\right), \ldots, \mathcal{M}^{\mathcal{E}}\left(t_{n}\right)\right), \\
\mathcal{M}_{v}^{\mathcal{E}}(A \wedge B)=\mathcal{M}_{v}^{\mathcal{E}}(A) \wedge \mathcal{M}_{v}^{\mathcal{E}}(B), \\
\mathcal{M}_{v}^{\mathcal{E}}(A \& B)=\mathcal{M}_{v}^{\mathcal{E}}(A) \otimes \mathcal{M}_{v}^{\mathcal{E}}(B) \\
\mathcal{M}_{v}^{\mathcal{E}}(A \equiv B)=\mathcal{M}_{v}^{\mathcal{E}}(A) \sim \mathcal{M}_{v}^{\mathcal{E}}(B) \\
\mathcal{M}_{v}^{\mathcal{E}}(\Delta A)=\Delta \mathcal{M}_{v}^{\mathcal{E}}(A) \\
\mathcal{M}_{v}^{\mathcal{E}}(\top)=\mathbf{1}, \quad \mathcal{M}_{v}^{\mathcal{E}}(\perp)=\mathbf{0} \\
\mathcal{M}_{v}^{\mathcal{E}}((\forall x) A)=\inf \left\{\mathcal{M}_{v^{\prime}}^{\mathcal{E}}(A) \mid v^{\prime}=v \backslash x\right\} \\
\mathcal{M}_{v}^{\mathcal{E}}((\exists x) A)=\sup \left\{\mathcal{M}_{v^{\prime}}^{\mathcal{E}}(A) \mid v^{\prime}=v \backslash x\right\}
\end{gathered}
$$

provided that the infimum (supremum) exists in the sense of $\mathcal{E}$; otherwise the truth value of the formula in question is undefined.

By $A_{x}[t]$ we denote a formula in which all free occurrences of a variable $x$ are replaced by the term $t$. A theory over predicate EQ-logic is a set $T$ of formulas. If $A$ is a formula then it is provable in $T$ if there is a proof of $A$. Then we write $T \vdash A$.

The structure $\mathcal{M}^{\mathcal{E}}$ is a model of $T, \mathcal{M}^{\mathcal{E}} \models T$, if $\mathcal{M}^{\mathcal{E}}(A)=\mathbf{1}$ holds for all axioms $A$ of $T$.

The structure $\mathcal{M}^{\mathcal{E}}$ is safe if all the needed infima and suprema exist, i.e. $\mathcal{M}_{v}^{\mathcal{E}}(A)$ is defined for all $A$ and each evaluation $v$ of variables.

A formula $A$ is a tautology if $\mathcal{M}_{v}^{\mathcal{E}}(A)=\mathbf{1}$ for each $\mathcal{E}$-safe structure $\mathcal{M}$ and each evaluation $v$. A formula $A$ is true in $T, T \models A$, if $\mathcal{M}_{v}^{\mathcal{E}}(A)=\mathbf{1}$ in all its models $\mathcal{M}^{\mathcal{E}}$.

The truth value of a formula $A$ in $\mathcal{E}$-structure $\mathcal{M}^{\mathcal{E}}$ is defined as follows:

$$
\mathcal{M}^{\mathcal{E}}(A)=\bigwedge\left\{\mathcal{M}_{v}^{\mathcal{E}}(A) \mid v \text { is an evaluation }\right\} .
$$

\subsection{Logical axioms and inference rules}

\section{Definition 7 (Logical axioms)}

The logical axioms of predicate EQ-logic are (EQ1)-

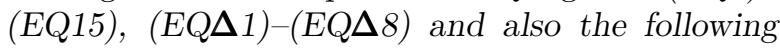
formulas:

$$
\begin{aligned}
& (E Q P 1)(\forall x) A(x) \Rightarrow A(t) \\
& \text { ( } t \text { substituable for } x \text { in } A(x) \text { ), }
\end{aligned}
$$

(EQP4) $(\forall x)(A \Rightarrow B) \Rightarrow((\exists x) A \Rightarrow B)$

$(x$ not free in $B)$,

$(E Q P 5)(\forall x)(A \vee B) \Rightarrow((\forall x) A \vee B)$

(x not free in $B)$.

\section{Remark 3}

The above axioms are the well known axioms on quantifiers used in many other kinds of predicate logics. Note that axiom (EQP3) is weakened by adding the $\Delta$-connective.

\section{Definition 8}

Basic inference rules of predicate EQ-logic are the Equanimity rule (EA), Leibniz rule

(Leib)

$$
\frac{A \equiv B}{C[\mathbf{p}:=A] \equiv C[\mathbf{p}:=B])},
$$

provided that the subformula $\mathbf{p}$ of $C$ is not in the scope of a quantifier in $C$, the necessitation rule $(\mathrm{N})$, and the rule of generalization

$$
\text { (G) } \frac{A}{(\forall x) A} \text {. }
$$

\subsection{Main properties}

\section{Lemma 5}

For an arbitrary formula $A, B, C$ where $x$ is not free in $A$, the following formulas are provable:
(a) $\vdash(A \Rightarrow(\forall x) B) \Rightarrow(\forall x)(A \Rightarrow B)$,
(b) $\vdash(\forall x)(B \Rightarrow A) \equiv((\exists x) B \Rightarrow A)$,
(c) $\vdash(\exists x)(A \Rightarrow B) \Rightarrow(A \Rightarrow(\exists x) B)$,
(d) $\vdash(\exists x)(B \Rightarrow A) \Rightarrow((\forall x) B \Rightarrow A)$,
(e) $\vdash \boldsymbol{\Delta}(\forall x)(C \Rightarrow B) \Rightarrow((\forall x) C \Rightarrow(\forall x) B)$,
(f) $\vdash(\forall x)(C \Rightarrow B) \Rightarrow((\exists x) C \Rightarrow(\exists x) B)$.

\section{Lemma 6}

If $A$ is an axiom of predicate $E Q_{\Delta}$-logic then for every structure $\mathcal{M}^{\mathcal{E}}$ and an evaluation $v, \mathcal{M}_{v}^{\mathcal{E}}(A)=$ 1 holds true.

PROOF: This is straightforward using the axioms and properties of $\ell \mathrm{EQ}_{\Delta}$-algebra.

\section{Lemma 7}

The inference rules of predicate $E Q_{\Delta}$-logic are sound, i.e. the following holds for every structure $\mathcal{M}^{\mathcal{E}}$ and every evaluation $v$ :

(a) If $\mathcal{M}_{v}^{\mathcal{E}}(A)=1$ and $\mathcal{M}_{v}^{\mathcal{E}}(A \equiv B)=1$ then $\mathcal{M}_{v}^{\mathcal{E}}(B)=\mathbf{1}$.

(b) If $\mathcal{M}_{v}^{\mathcal{E}}(B \equiv C)=\mathbf{1}$ then $\mathcal{M}_{v}^{\mathcal{E}}(A[\mathbf{p}:=B] \equiv$ $A[\mathbf{p}:=C])=\mathbf{1}$ for any formula $A$.

(c) If $\mathcal{M}_{v}^{\mathcal{E}}(A)=\mathbf{1}$ then $\mathcal{M}_{v}^{\mathcal{E}}(\Delta A)=\mathbf{1}$.

(d) If $\mathcal{M}_{v}^{\mathcal{E}}(A)=\mathbf{1}$ then $\mathcal{M}_{v}^{\mathcal{E}}((\forall x) A)=\mathbf{1}$.

PROOF:

(a) Obviously, if $a=1$ and $a \sim b=1$ then necessarily $b=1$.

(b) By induction on the complexity of the formula $A$. If $A$ is $\mathbf{p}$ then $\mathcal{M}_{v}^{\mathcal{E}}(A[\mathbf{p}:=B] \equiv A[\mathbf{p}:=C])=$ $\mathcal{M}_{v}^{\mathcal{E}}(B \equiv C)=\mathbf{1}$. If $A$ is $\top, \perp, P\left(t_{1}, \ldots, t_{n}\right)$ or $\mathbf{q}$ (other than $\mathbf{p}$ ) then $\mathcal{M}_{v}^{\mathcal{E}}(A[\mathbf{p}:=B] \equiv A[\mathbf{p}:=$ $C])=\mathcal{M}_{v}^{\mathcal{E}}(A \equiv A)=\mathbf{1}$.

For induction step, we pick an arbitrary nonatomic $A$ and prove

$$
\mathcal{M}_{v}^{\mathcal{E}}(A[\mathbf{p}:=B] \equiv A[\mathbf{p}:=C])=\mathbf{1}
$$


that is

$$
\mathcal{M}_{v}^{\mathcal{E}}(A[\mathbf{p}:=B]) \sim \mathcal{M}_{v}^{\mathcal{E}}(A[\mathbf{p}:=C])=\mathbf{1}
$$

and thus with the using of property (1) and this that every good algebra is also separated conclude

$$
\mathcal{M}_{v}^{\mathcal{E}}(A[\mathbf{p}:=B])=\mathcal{M}_{v}^{\mathcal{E}}(A[\mathbf{p}:=C])
$$

on the induction hypothesis (I.H.) that the claim $\mathcal{M}_{v}^{\mathcal{E}}(D[\mathbf{p}:=B])=\mathcal{M}_{v}^{\mathcal{E}}(D[\mathbf{p}:=C])$ is true for all formulae less complex than $A$.

We have this case: Let $A$ be $E \wedge F$. The I.H. applies on $E$ and $F$.

Now, $A[\mathbf{p}:=B] \equiv A[\mathbf{p}:=C])$ is $\mathcal{M}_{v}^{\mathcal{E}}(E[\mathbf{p}:=$ $B] \wedge F[\mathbf{p}:=B] \equiv F[\mathbf{p}:=C] \wedge F[\mathbf{p}:=C])$ thus we get (5) as follows:

$$
\begin{gathered}
\mathcal{M}_{v}^{\mathcal{E}}(E[\mathbf{p}:=B] \wedge F[\mathbf{p}:=B])= \\
\mathcal{M}_{v}^{\mathcal{E}}(E[\mathbf{p}:=B]) \wedge \mathcal{M}_{v}^{\mathcal{E}}(F[\mathbf{p}:=B])= \\
\left.\mathcal{M}_{v}^{\mathcal{E}}(E[\mathbf{p}:=C]) \wedge \mathcal{M}_{v}^{\mathcal{E}}(F[\mathbf{p}:=C]) \text { (by I.H. }\right)= \\
\mathcal{M}_{v}^{\mathcal{E}}(E[\mathbf{p}:=C] \wedge F[\mathbf{p}:=C])
\end{gathered}
$$

The cases where $A$ is $E \& F, E \vee F$ or $E \equiv F$ are proved analogously.

Let $A$ be $(\forall x) E$. Then

$$
\begin{gathered}
\left.\mathcal{M}_{v}^{\mathcal{E}}((\forall x) E[\mathbf{p}:=B])\right)= \\
\inf \left\{\mathcal{M}_{v^{\prime}}^{\mathcal{E}}\left((E[\mathbf{p}:=B]) \mid v^{\prime}=v \backslash x\right\}=\right. \\
\inf \left\{\mathcal{M}_{v^{\prime}}^{\mathcal{E}}\left((E[\mathbf{p}:=C]) \mid v^{\prime}=v \backslash x\right\}=\right. \\
\left.\mathcal{M}_{v}^{\mathcal{E}}((\forall x) E[\mathbf{p}:=C])\right) .
\end{gathered}
$$

The case where $A$ is $(\exists x) E$ can be proved by the similar way.

Let $A$ be $\Delta E$. Then

$$
\begin{gathered}
\left.\left.\mathcal{M}_{v}^{\mathcal{E}}(\Delta E[\mathbf{p}:=B])\right)=\Delta \mathcal{M}_{v}^{\mathcal{E}}(E[\mathbf{p}:=B])\right)= \\
\left.\left.\Delta \mathcal{M}_{v}^{\mathcal{E}}(E[\mathbf{p}:=C])\right)=\mathcal{M}_{v}^{\mathcal{E}}(\Delta E[\mathbf{p}:=C])\right)
\end{gathered}
$$

(c) is trivial. (d) is obvious from definition $\mathcal{M}^{\mathcal{E}}(A)$ (see (4)).

The following is the deduction theorem formulated in the style natural for EQ-logic. The proof proceeds by induction on the length of the proof of $C$.

\section{Theorem 5}

For each theory $T$, closed formulas $A, B$ and arbitrary formula $C$ it holds that

$$
T \cup\{A \equiv B\} \vdash C \quad \text { iff } \quad T \vdash \Delta(A \equiv B) \Rightarrow C .
$$

If we put $B:=\top$ in Theorem 5 then we get the "standard" form of the delta deduction theorem.

\section{Corollary 1}

For each theory $T$, closed formula $A$ and arbitrary formula $C$ it holds that

$$
T \cup\{A\} \vdash C \quad \text { iff } \quad T \vdash \Delta A \Rightarrow C .
$$

\section{Definition 9}

Let $T$ be a theory. Then we say that

(a) $T$ is inconsistent if $T \vdash \perp$. Otherwise it is consistent.

(b) $T$ is maximal consistent if each its extension $S$, $T \subset S$ is contradictory.

(c) $T$ is linear*) if

$$
T \vdash A \Rightarrow B \quad \text { or } \quad T \vdash B \Rightarrow A
$$

for every two formulas $A, B$.

(d) $T$ is extensionally complete if for every formula of the form $(\forall x)(A(x) \equiv B(x))$ such that $T \forall$ $(\forall x)(A(x) \equiv B(x))$ there is a constant $\mathbf{u}$ for which $T \forall\left(A_{x}[\mathbf{u}] \equiv B_{x}[\mathbf{u}]\right)$.

\subsection{Completeness}

The proofs of the following two theorems proceed similarly as items 3 and 1 of [9, Lemma 2].

\section{Theorem 6}

Every consistent theory $T$ can be extended to a maximally consistent linear theory.

\section{Theorem 7}

Every consistent theory $T$ can be extended to an extensionally complete consistent theory $T^{\prime}$.

Now we construct the Lindenbaum algebra $\mathcal{E}_{T}$ for the theory $T$ in a standard way from equivalence classes using the following equivalence on formulas:

$$
A \approx B \quad \text { iff } \quad T \vdash A \equiv B, \quad A, B \in F_{J} .
$$

\section{Theorem 8}

Let $T$ be a linear extensionally complete theory. Then the algebra

$$
\mathcal{E}_{T}=\left\langle\bar{E}, \wedge_{T}, \vee_{T}, \otimes_{T}, \sim_{T}, \Delta_{T}, \mathbf{0}_{T}, \mathbf{1}_{T}\right\rangle
$$

is a non-commutative linearly ordered $\ell E Q_{\Delta^{-}}$ algebra.

PROOF: It is easy to verify the axioms of noncommutative linearly ordered $\ell \mathrm{EQ}_{\Delta}$-algebra. Moreover,

$$
\begin{gathered}
|A| \leq|B| \quad \text { iff }|A| \wedge_{T}|B|=|A| \\
\text { iff } \quad T \vdash(A \wedge B) \equiv A \text { iff } T \vdash A \Rightarrow B \\
\text { iff } T \vdash(A \Rightarrow B) \equiv \top \text { iff }|A| \rightarrow_{T}|B|=|\top| .
\end{gathered}
$$

From here and the linearity of $T$ follow that $\mathcal{E}_{T}$ is linear ordered.

Analogously as in Lemma 5.2.6 from [8] we prove the following equalities:

$$
\begin{aligned}
& |(\forall x) A|=\bigwedge\left\{\left|A_{x}[\mathbf{u}]\right| \mid \text { all constants } \mathbf{u} \in \text { Const }\right\} \\
& |(\exists x) A|=\bigvee\left\{\left|A_{x}[\mathbf{u}]\right| \mid \text { all constants } \mathbf{u} \in \text { Const }\right\} .
\end{aligned}
$$

\footnotetext{
*) In [8] and elsewhere such a theory is called complete.
} 


\section{Definition 10}

Let $T$ be a linear extensionally complete theory. Then the canonical model of the theory $T$ is the following structure:

$$
\mathcal{M}^{T}=\left\langle M, \mathcal{E}_{T},\left\{r_{P}\right\}_{P \in \text { Pred }},\left\{m_{u}\right\}_{u \in \text { Const }}\right\rangle,
$$

where we put $M$ as the set of all constants of the language of $T, \mathcal{E}_{T}$ is Lindenbaum algebra (8), $m_{\mathbf{u}}=$ $\mathbf{u}$ for each such constant and $r_{P}=\left|P\left(u_{1}, \ldots, u_{n}\right)\right|_{T}$.

Now, we prove the following version of completeness of predicate EQ-logic.

\section{Theorem 9}

A theory $T$ is consistent iff it has a safe model $\mathcal{M}$.

PROOF: Suppose that $T$ is inconsistent. This means that $T \vdash \perp$. Thus, if $\mathcal{M} \models T$ then $\mathcal{M}_{v}(\perp)=$ 1 that is impossible.

For the proof of the converse implication, we expand $T$ to a linear extensionally complete theory $\bar{T}$. Further, we construct its canonical model $\mathcal{M}^{T}$. Assume that $A$ is an axiom of $T$, thus $T \vdash A$ and $\bar{T} \vdash A$ too. Using (EQ1) and rule (EA) we obtain $\bar{T} \vdash A \equiv T$, thus $T \vdash A \equiv T$ and consequently $\mathcal{M}_{v}^{T}(A)=|\top|=\mathbf{1}_{T}$. Hence, $\mathcal{M}^{T}$ is a model of $T$.

\section{Theorem 10}

Let $T$ be a theory. Then for each formula $A$

$$
T \vdash A \quad \text { iff } \quad T \models A .
$$

PROOF: The implication left-to-right follows from Lemma (6) and (7).

For the converse implication, assume that $T \forall A$. We have to show that it exists a model $\mathcal{M}$ of a theory $T$ and evaluation $v$ such that $\mathcal{M}_{v}(A) \neq \mathbf{1}$. Let us take the canonical model $\mathcal{M}^{T}$ of the theory $T$ and let $\mathcal{M}_{v}^{T}(A)=\mathbf{1}=|\top|$ for some evaluation $v$. Thus, $T \vdash A \equiv \top$, and so, $T \vdash A$. Therefore, $T \nvdash A$ means that $\mathcal{M}_{v}^{T}(A) \neq \mathbf{1}_{T}$.

\section{Conclusion}

After propositional and higher order EQ-logic, this paper deals with the third kind of it, namely the first-order EQ-logic. By this, we concluded the basic phase of the development of logics, whose truth values are formed by an EQ-algebra. This algebra is characteristic by taking fuzzy equality as the basic operation and implication is derived from it. Moreover, the multiplication (serving as natural interpretation of strong conjunction) is in general noncommutative. EQ-algebras algebra generalize residuated lattices in the sense that every residuated lattice is an EQ-algebra but not vice-versa.

After detailed analysis it turned out that first-order EQ-logic requires presence of the $\Delta$ connective. The reason is that classical properties of the fuzzy equality must be preserved in limit cases. Moreover, the EQ-algebra used as an algebra of truth values must be linearly ordered. Using standard techniques adapted for specific properties of EQ-algebra, we proved that the first-order EQlogic is syntactico-semantically complete.

\section{Acknowledgment}

This work was supported by the European Regional Development Fund in the IT4Innovations Centre of Excellence project (CZ.1.05/1.1.00/02.0070).

\section{References}

[1] M. Dyba and V. Novák. EQ-logics: Noncommutative fuzzy logics based on fuzzy equality. Fuzzy Sets and Systems, 172:13-32, 2011.

[2] V. Novák. EQ-algebra-based fuzzy type theory and its extensions. Logic Journal of the IGPL, 19:512-542, 2011.

[3] V. Novák. EQ-algebras: primary concepts and properties. In Proc. Czech-Japan Seminar, Ninth Meeting. Kitakyushu\& Nagasaki, August 18-22, 2006, pages 219-223. Graduate School of Information, Waseda University, 2006.

[4] M. El-Zekey, V. Novák, and R. Mesiar. On good EQ-algebras. Fuzzy Sets and Systems, 178:1-23, 2011.

[5] V. Novák and B. de Baets. EQ-algebras. Fuzzy Sets and Systems, 160:2956-2978, 2009.

[6] M. El-Zekey. Representable good EQ-algebras. Soft Computing, 14:1011-1023, 2009.

[7] M. Dyba and V. Novák. EQ-logics with delta connective. Iranian Journal of Fuzzy Systems, (submitted).

[8] P. Hájek. Metamathematics of Fuzzy Logic. Kluwer, Dordrecht, 1998.

[9] P. Hájek and P. Cintula. On theories and models in fuzzy predicate logics. Journal of Symbolic Logic, 71(3):863Ü-880, 2006. 